\title{
DISCOVERY OF VERY HIGH ENERGY $\gamma$-RAY EMISSION FROM THE LOW-FREQUENCY-PEAKED BL LACERTAE OBJECT BL LACERTAE
}

J. Albert, ${ }^{1}$ E. Aliu, ${ }^{2}$ H. Anderhub,${ }^{3}$ P. Antoranz,${ }^{4}$ A. Armada,${ }^{2}$ C. Baixeras,${ }^{5}$ J. A. Barrio, ${ }^{4}$ H. Bartko, ${ }^{6}$ D. Bastieri,${ }^{7}$ J. K. Becker, ${ }^{8}$ W. Bednarek, ${ }^{9}$ K. Berger,${ }^{1}$ C. Bigongiari,${ }^{7}$ A. Biland, ${ }^{3}$ R. K. Bock,${ }^{6,7}$ P. Bordas, ${ }^{10}$ V. Bosch-Ramon, ${ }^{10}$

T. Bretz,${ }^{1}$ I. Britvitch, ${ }^{3}$ M. Camara,${ }^{4}$ E. Carmona,${ }^{6}$ A. Chilingarian, ${ }^{11}$ J. A. Coarasa,${ }^{6}$ S. Commichau, ${ }^{3}$

J. L. Contreras, ${ }^{4}$ J. Cortina,${ }^{2}$ M. T. Costado, ${ }^{12}$ V. Curtef,${ }^{8}$ V. Danielyan,,${ }^{11}$ F. Dazzi, ${ }^{7}$ A. De Angelis,${ }^{13}$ C. Delgado, ${ }^{12}$ R. De los Reyes, ${ }^{4}$ B. De Lotto, ${ }^{13}$ E. Domingo-Santamaría, ${ }^{2}$ D. Dorner, ${ }^{1}$ M. Doro, ${ }^{7}$ M. Errando, ${ }^{2}$ M. Fagiolini,,${ }^{14}$

D. Ferenc, ${ }^{15}$ E. Fernández, ${ }^{2}$ R. Firpo, ${ }^{2}$ J. Flix,${ }^{2}$ M. V. Fonseca, ${ }^{4}$ L. Font,${ }^{5}$ M. Fuchs,${ }^{6}$ N. Galante, ${ }^{6}$ R. García-López,${ }^{12}$ M. GarczarczyK, ${ }^{6}$ M. Gaug,${ }^{7}$ M. Giller, ${ }^{9}$ F. Goebel,${ }^{6}$ D. Hakobyan, ${ }^{11}$ M. Hayashida,,${ }^{6,16}$ T. Hengstebeck,${ }^{17}$ A. Herrero, ${ }^{12}$ D. Höhne, ${ }^{1}$ J. Hose,${ }^{6}$ C. C. Hsu,${ }^{6}$ P. Jacon, ${ }^{9}$ T. Jogler, ${ }^{6}$ R. Kosyra,${ }^{6}$ D. Kranich, ${ }^{3}$ R. Kritzer,${ }^{1}$ A. Laille, ${ }^{15}$ E. Lindfors,${ }^{18}$ S. Lombardi,${ }^{7}$ F. Longo, ${ }^{13}$ J. López,${ }^{2}$ M. López,${ }^{4}$ E. Lorenz,${ }^{3,6}$ P. Majumdar,${ }^{6}$ G. Maneva ${ }^{19}$ K. Mannheim, ${ }^{1}$ O. Mansutti,${ }^{13}$ M. Mariotti,${ }^{7}$ M. Martínez, ${ }^{2}$ D. Mazin,,${ }^{6}$ C. Merck, ${ }^{6}$ M. Meucci,${ }^{14}$ M. Meyer, ${ }^{1}$ J. M. Miranda, ${ }^{4}$ R. Mirzoyan, ${ }^{6}$ S. Mizobuchi,${ }^{6}$ A. Moralejo, ${ }^{2}$ K. Nilsson,${ }^{18}$ J. Ninkovic, ${ }^{6}$ E. OÑa-Wilhelmi, ${ }^{2}$ N. Otte,${ }^{6,17}$ I. Oya,${ }^{4}$ D. Paneque,${ }^{6}$ M. Panniello,${ }^{12}$ R. Paoletti,${ }^{14}$ J. M. Paredes,${ }^{10}$ M. Pasanen, ${ }^{18}$ D. Pascoli,${ }^{7}$ F. Pauss, ${ }^{3}$ R. Pegna,${ }^{14}$ M. Persic,${ }^{13,20}$ L. Peruzzo,${ }^{7}$ A. Piccioli,${ }^{14}$ M. Poller,${ }^{1}$ E. Prandini,${ }^{7}$ N. Puchades,${ }^{2}$ A. Raymers,${ }^{11}$ W. Rhode, ${ }^{8}$ M. Ribó, ${ }^{10}$ J. Rico, ${ }^{2}$ M. Rissi,${ }^{3}$ A. Robert, ${ }^{5}$ S. RÜGamer, ${ }^{1}$ A. Saggion, ${ }^{7}$ A. Sánchez, ${ }^{5}$ P. Sartori,${ }^{7}$ V. Scalzotto, ${ }^{7}$ V. Scapin, ${ }^{13}$ R. Schmitt, ${ }^{1}$ T. Schweizer,${ }^{6}$ M. Shayduk,${ }^{6,17}$ K. Shinozaki,${ }^{6}$ S. N. Shore, ${ }^{21}$ N. Sidro, ${ }^{2}$ A. Sillanpää, ${ }^{18}$ D. Sobczynska, ${ }^{9}$ A. Stamerra, ${ }^{14}$ L. S. Stark, ${ }^{3}$ L. Takalo, ${ }^{18}$ P. Temnikov,,${ }^{19}$ D. Tescaro, ${ }^{2}$ M. Teshima, ${ }^{6}$ N. Tonello,${ }^{6}$ D. F. Torres, ${ }^{2,22}$ N. Turini,${ }^{14}$ H. Vankov,,${ }^{19}$ V. Vitale,${ }^{13}$ R. M. Wagner,${ }^{6}$ T. Wibig, ${ }^{9}$ W. WitteK, ${ }^{6}$

F. ZANDANEL, ${ }^{7}$ R. ZANin, ${ }^{2}$ AND J. ZAPATERO ${ }^{5}$

Received 2007 March 5; accepted 2007 July 11; published 2007 August 16

\section{ABSTRACT}

The MAGIC collaboration observed BL Lacertae for $22.2 \mathrm{hr}$ during 2005 August to December and for $26 \mathrm{hr}$ during 2006 July to September. The source is the historical prototype and eponym of a class of low-frequency-peaked BL Lacertae (LBL) objects. A very high energy (VHE) $\gamma$-ray signal was discovered with a $5.1 \sigma$ excess in the 2005 data. Above $200 \mathrm{GeV}$, an integral flux of $(0.6 \pm 0.2) \times 10^{-11} \mathrm{~cm}^{-2} \mathrm{~s}^{-1}$ was measured, corresponding to approximately $3 \%$ of the Crab flux. The differential spectrum between 150 and $900 \mathrm{GeV}$ is rather steep with a photon index of $-3.6 \pm$ 0.5 . The light curve shows no significant variability during the observations in 2005. For the first time a clear detection of VHE $\gamma$-ray emission from an LBL object was obtained with a signal below previous upper limits. The 2006 data show no significant excess. This drop in flux follows the observed trend in optical activity.

Subject headings: BL Lacertae objects: individual (BL Lacertae) — gamma rays: observations

Online material: color figures

\section{INTRODUCTION}

BL Lacertae (1ES 2200+420, $z=0.069$; Miller et al. 1978) is the historical prototype of a class of powerful $\gamma$-ray emitters: "BL Lac objects." It belongs to an active galactic nucleus (AGN)

\footnotetext{
${ }^{1}$ Universität Würzburg, D-97074 Würzburg, Germany.

${ }^{2}$ Institut de Física d'Altes Energies, E-08193 Bellaterra, Spain.

${ }^{3}$ ETH Zurich, CH-8093 Zurich, Switzerland.

${ }^{4}$ Universidad Complutense, E-28040 Madrid, Spain.

${ }^{5}$ Universitat Autònoma de Barcelona, E-08193 Bellaterra, Spain.

${ }^{6}$ Max-Planck-Institut für Physik, D-80805 Munich, Germany.

${ }^{7}$ Università di Padova and INFN, I-35131 Padova, Italy.

${ }^{8}$ Universität Dortmund, D-44227 Dortmund, Germany.

${ }^{9}$ University of Łódź, PL-90236 Lodz, Poland.

${ }^{10}$ Universitat de Barcelona, E-08028 Barcelona, Spain.

${ }^{11}$ Yerevan Physics Institute, AM-375036 Yerevan, Armenia.

${ }^{12}$ Instituto de Astrofisica de Canarias, E-38200 La Laguna, Tenerife, Spain.

${ }^{13}$ Università di Udine and INFN Trieste, I-33100 Udine, Italy.

${ }^{14}$ Università di Siena and INFN Pisa, I-53100 Siena, Italy.

${ }^{15}$ University of California, Davis, CA 95616-8677.

${ }^{16}$ Corresponding author; mahaya@mppmu.mpg.de.

${ }^{17}$ Humboldt-Universität zu Berlin, D-12489 Berlin, Germany.

${ }^{18}$ Tuorla Observatory, Turku University, FI-21500 Piikkiö, Finland.

${ }^{19}$ Institute for Nucl. Research and Nucl. Energy, BG-1784 Sofia, Bulgaria.

${ }^{20}$ INAF-Osservatorio Astronomico and INFN Trieste, I-34131 Trieste, Italy.

${ }^{21}$ Università di Pisa and INFN Pisa, I-56126 Pisa, Italy.

${ }^{22}$ ICREA and Inst. Cienciès l'Espai, IEEC-CSIC, E-08193 Bellaterra, Spain.
}

subclass in which the jet is aligned very close to our line of sight. The mass of the supermassive black hole in the center is estimated to be $\sim 10^{8} M_{\odot}$ (Wu et al. 2002). Electromagnetic emission from this class of sources can be observed from the radio up to very high energy (VHE) $\gamma$-rays $(E>100 \mathrm{GeV})$, with spectral energy distributions (SEDs) characterized by a two-bump structure. So far, most of the measured SEDs could be interpreted using scenarios of a simple leptonic origin. The lower energy bump is produced by synchrotron radiation of relativistic electrons, while the higher energy bump originates from inverse Compton (IC) scattering of the same electron population accelerated in the jet. The target photons for IC scattering could be either synchrotron photons (synchrotron self-Compton scattering, SSC) or external photons either from the broad-line emission region or from the accretion disk (external inverse Compton scattering, EC). If both photon populations contribute to the IC emission, the corresponding IC component may have a double local-peak structure (e.g., Ghisellini et al. 1998). When the synchrotron emission peak is located in the submillimeter to optical band, the objects are classified as "low-frequency-peaked BL Lac objects" (LBLs), whereas in "high-frequency-peaked BL Lac objects" (HBLs) the synchrotron peak is located at UV to X-ray energies (Padovani \& Giommi 1995; Fossati et al. 1998). BL Lac objects often show a strong 
flux variability down to timescales of a few minutes for the highest energy range (Gaidos et al. 1996; Albert et al. 2007c). It should be noted that other models, based, e.g., on the acceleration of hadrons (Mannheim 1993; Mücke \& Protheroe 2001), could also explain the SEDs of BL Lac objects.

Up to now, there are 13 well-established extragalactic VHE $\gamma$ ray sources. Twelve are classified as HBLs and one is the FanaroffRiley class I radio galaxy, M87. BL Lacertae is classified as an LBL object with a synchrotron peak frequency of $2.2 \times 10^{14} \mathrm{~Hz}$ (Sambruna et al. 1999), and is one of the best-studied objects in the various energy bands. In 1998 and 2001, VHE $\gamma$-ray detection of the LBLs 3C 66A and BL Lacertae were claimed (Neshpor et al. 1998, 2001). Subsequent observations with more sensitive instruments have not, however, revealed any evidence for VHE $\gamma$ ray emission consistent with these claims. No VHE $\gamma$-ray emission from any LBLs has previously been confirmed.

Villata et al. (2004) have presented long-term light curves in optical and radio emission over 30 years and reported cross-correlation between the optical light curve and radio hardness ratio with some delay in radio emission. They have claimed evidence of an $\sim 8$ yr periodicity in radio but less evidence in optical. Several authors (e.g., Hagen-Thorn et al. 1997; Stirling et al. 2003) have also reported periodic and quasi-periodic variations in the optical and radio light curves.

Gamma-ray observations by EGRET resulted only in an upper limit of $1.4 \times 10^{-7} \mathrm{~cm}^{-2} \mathrm{~s}^{-1}$ until 1995. Later, EGRET observed $\gamma$-rays above $100 \mathrm{MeV}$ at a flux level of $(4.0 \pm$ 1.2) $\times 10^{-7} \mathrm{~cm}^{-2} \mathrm{~s}^{-1}$ with $4.4 \sigma$ significance (Catanese et al. 1997). During an optical outburst in 1997, a $\gamma$-ray flare was measured with $10 \sigma$ significance by EGRET at a flux level of $(1.72 \pm 0.42) \times 10^{-6} \mathrm{~cm}^{-2} \mathrm{~s}^{-1}, 12$ times higher than the previous upper limit (Bloom et al. 1997). The EC, in which the broad-line emission flux has been Comptonized, has been suggested for the interpretation of the $\gamma$-ray emission in the 1997 flare (e.g., Madejski et al. 1999; Ravasio et al. 2002).

In the VHE $\gamma$-ray range, the Crimean Observatory has claimed a detection with $7.2 \sigma$ significance (Neshpor et al. 2001), while HEGRA, observing in the same period, obtained only a much lower upper limit (Kranich 2003; see details in § 4). Other past observations of this target resulted in upper limits only (Catanese et al. 1997; Kranich 2003; Aharonian et al. 2004; Horan et al. 2004).

In this Letter we report about the discovery of VHE $\gamma$-ray emission from BL Lacertae in 2005. Simultaneous observation in the optical band in 2005 and 2006 permitted a search for correlations between the optical and VHE $\gamma$-ray activities.

\section{OBSERVATIONS AND DATA ANALYSIS}

BL Lacertae (R.A. $22^{\mathrm{h}} 02^{\mathrm{m}} 43.3^{\mathrm{s}}$, decl. $+42^{\circ} 16^{\prime} 40^{\prime \prime}$; J2000.0) was observed with the $17 \mathrm{~m}$ diameter MAGIC (Major Atmospheric Gamma Imaging Cerenkov) telescope on the Canary Island of La Palma (N28.2 ${ }^{\circ}, \mathrm{W} 17.8^{\circ}, 2225 \mathrm{~m}$ a.s.l.). The MAGIC telescope is the world's largest imaging atmospheric Cerenkov telescope (IACT). The telescope parameters and performance are described in Baixeras et al. (2004) and Cortina et al. (2005). The source was observed for $22.2 \mathrm{hr}$ from 2005 August until December. The telescope was pointing directly onto the object, recording so-called $\mathrm{ON}$ data. The background was estimated from observations of regions where no $\gamma$-rays are expected, which we define as OFF data, which were taken with sky conditions similar to those of $\mathrm{ON}$ data.

The raw data were first calibrated (Gaug et al. 2005), then processed with the standard MAGIC analysis and reconstruction software (Bretz et al. 2005). Data runs with anomalous trigger rates due to bad observation conditions were rejected from further analysis. The remaining ON data corresponded to $17.8 \mathrm{hr}$, while the OFF data corresponded to $57.2 \mathrm{hr}$. Image parameters of the raw data (Hillas 1985) were calculated and compared for the ON and OFF data to check their consistency; excellent agreement was found. The selected data samples before the $\gamma /$ hadron separation are completely dominated by hadron events, and the expected admixture of $\gamma$-ray events are below the statistical fluctuations of the data.

In order to reject most hadronic background while still conserving the majority of the $\gamma$-candidates, a multitree classifier algorithm based on the "random forest" (RF) method (Breiman 2001; Bock et al. 2004) was used for the $\gamma /$ hadron separation. The selection conditions were trained with Monte Carlo simulated $\gamma$-ray samples (Knapp \& Heck 2004; Majumdar et al. 2005) and a sample of experimental background events. In the RF method for each event the so-called HADRONNESS parameter $(\mathrm{H})$ was calculated from a combination of all the image parameters except the ALPHA parameter. ALPHA is the angle between the shower image principal axis and the line connecting the image center of gravity with the camera center. $\mathrm{H}$ assigns to each event a number between 0 and 1 of being more hadron-like (high $\mathrm{H}$ ) or $\gamma$-ray like (low $\mathrm{H}$ values). The selection of events with low $\mathrm{H}$ value enriches $\gamma$-ray events in the surviving data sample. Finally, the image parameter ALPHA was calculated and the ALPHA distribution for the $\mathrm{ON}$ and OFF data compared after normalizing them on the area between $20^{\circ}$ and $80^{\circ}$. Any $\gamma$-ray signal should show up as an excess at small ALPHA values. The number of background events in the ON distribution at small ALPHA was determined by a secondorder polynomial fit (without linear term) to the ALPHA distribution of the normalized OFF data. For our analysis we used an ALPHA cut of $9^{\circ}$ (see also Albert et al. 2006, 2007a, 2007b).

The cuts in H and ALPHA were optimized using data samples from Crab Nebula observations at comparable zenith angles. The significance of an excess visible in the ALPHA distribution was calculated according to equation (17) in Li \& Ma (1983). The energy of the $\gamma$-ray events was also reconstructed by means of the RF method with MC $\gamma$-samples. The average energy resolution is estimated to be about $24 \% \mathrm{rms}$.

Optical $R$-band observations were provided by the Tuorla Observatory Blazar Monitoring Program ${ }^{23}$ with the 1.03 m telescope at the Tuorla Observatory, Finland, and the $35 \mathrm{~cm}$ KVA telescope on La Palma, Canary Islands. The magnitudes were then converted to linear fluxes using the formula $F(\mathrm{Jy})=3080.0 \times 10^{-(\mathrm{mag} / 2.5)}$.

Radio observations were also performed with $\mathrm{UMRAO}^{24}$ at 4.8, 8.0, and $14.5 \mathrm{GHz}$, and at $37 \mathrm{GHz}$ with the Metsähovi Radio Observatory.

\section{RESULTS}

Figure 1 shows the local sky map for the $2005 \gamma$-ray candidates using the DISP method (Lessard et al. 2001). Only events $>350$ photoelectrons were used, corresponding to an energy threshold of about $200 \mathrm{GeV} \gamma$-ray energy. The map was produced from the excess events distribution smoothed with a 2D Gaussian of $0.1^{\circ}$. The black cross marks the nominal position of BL Lacertae. The small offset and the extension of the image are comparable to the telescope PSF $\left(0.1^{\circ}\right)$ and the telescope pointing error $\left(1.5^{\prime}\right)$.

In Figure 2, the ALPHA distribution is shown. An excess of 216 events over 1275.6 normalized background events yields a significance of $5.1 \sigma$ for data above 350 photoelectrons. Two mostly independent analyses confirmed the result.

The 2005 VHE $\gamma$-ray and the 2005 and 2006 optical light curves are shown in Figure 3. No significant evidence of flux variability

\footnotetext{
${ }^{23}$ More information at http://users.utu.fi/kani/1m.

${ }^{24}$ UMRAO is partially supported by a series of grants from the NSF and by funds from the University of Michigan.
} 


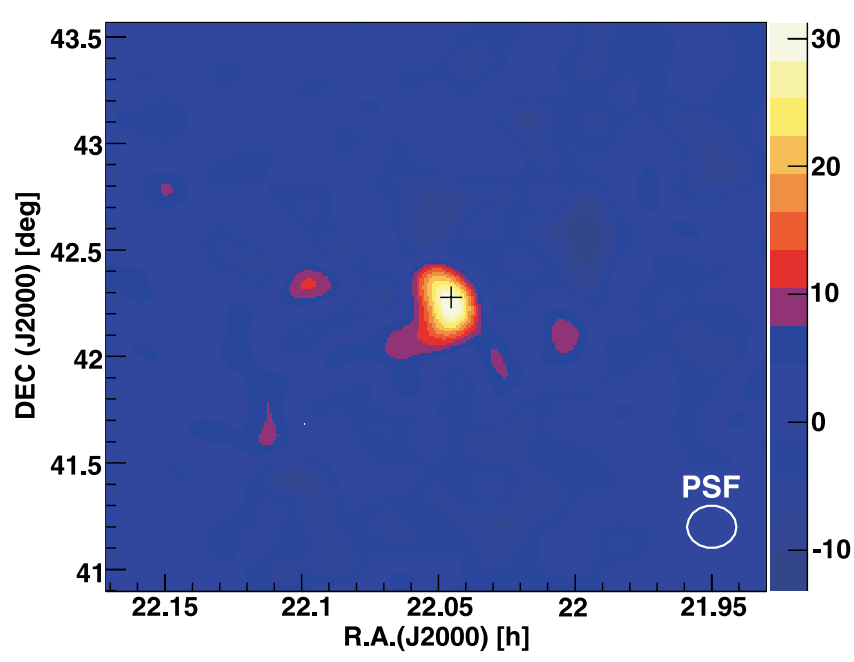

FIG. 1.-Sky map of the region around the position of BL Lacertae (cross) for reconstructed $\gamma$-events with $>350$ photoelectrons (corresponding to an energy threshold of about $200 \mathrm{GeV}$ ) from the 2005 observations.

in VHE $\gamma$-rays was found in the 2005 data. The derived average integral flux is $F(E>200 \mathrm{GeV})=(0.6 \pm 0.2) \times 10^{-11} \mathrm{~cm}^{-2}$ $\mathrm{s}^{-1}\left(\chi^{2} /\right.$ dof $\left.=16.3 / 15\right)$, which corresponds to about $3 \%$ of the Crab Nebula flux as measured by the MAGIC telescope (Wagner et al. 2005). In the optical light curve, the contribution from the host galaxy (1.38 mJy) was subtracted. The optical light curve shows a flare around the end of 2005 October (MJD 53,670). Also radio light curves at $37 \mathrm{GHz}$ (A. Lähteemäki 2006, private communication) and $14.5 \mathrm{GHz}$ (UMRAO) suggest some minor flaring activity starting in 2005 November.

The reconstructed differential energy spectrum (Fig. 4) is well described by a simple power law:

$$
\frac{d N}{d E}=(1.9 \pm 0.5) \times 10^{-11}\left(\frac{E}{300 \mathrm{GeV}}\right)^{-3.6 \pm 0.5} \frac{\gamma}{\mathrm{TeV} \mathrm{s} \mathrm{cm}}{ }^{2}
$$

Because of the relatively steep slope, the systematic errors are estimated to be $\sim 50 \%$ for the absolute flux level and 0.2 for the spectral index.

Follow-up observations were carried out from 2006 July to

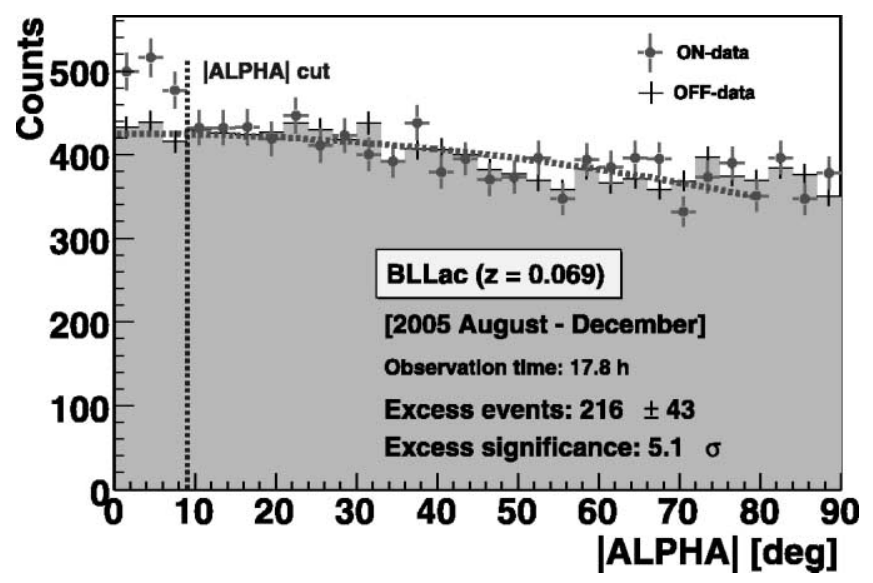

FIG. 2.-ALPHA-distribution of the 2005 data. The filled circles represent ON data. The light crosses correspond to normalized OFF data, and a dotted curve describes a second-order polynomial fit to the distribution of the OFF data. The vertical line indicates the ALPHA selection condition, which yields a total excess of 216 events at a significance level of $5.1 \sigma$. [See the electronic edition of the Journal for a color version of this figure.]
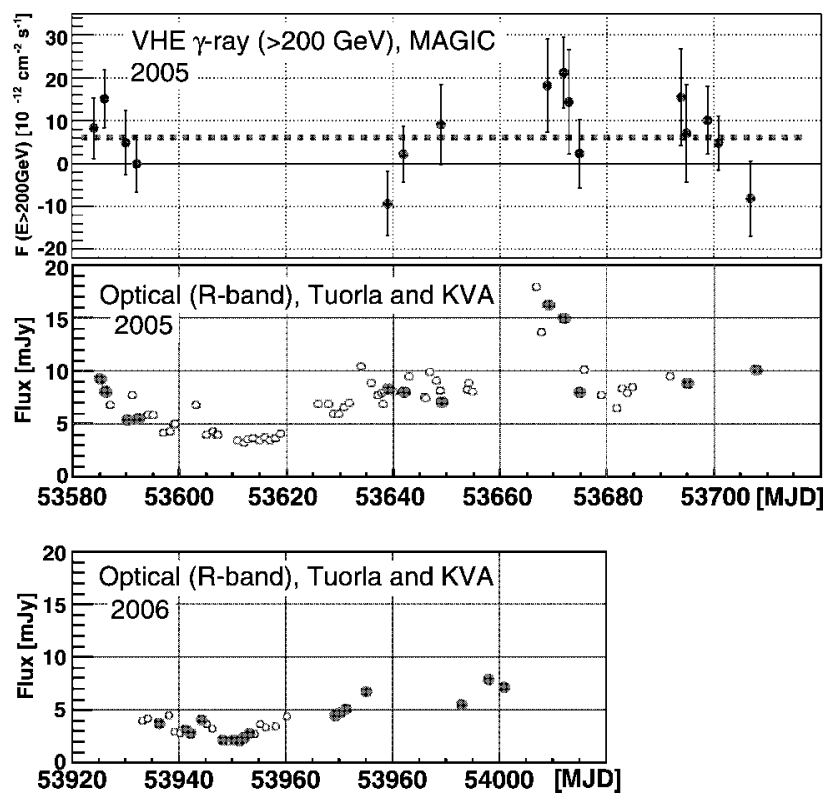

FIG. 3.- Light curve of VHE $\gamma$-ray $(>200 \mathrm{GeV})$ flux as measured with the MAGIC telescope during 2005 (top). Dotted horizontal line represents the average flux. Light curve of optical ( $R$ band) flux during 2005 (middle) and 2006 (bottom) as measured with the $1.03 \mathrm{~m}$ Tuorla and the $35 \mathrm{~cm}$ KVA telescopes. In the optical light curve, filled points represent the optical flux when simultaneous MAGIC observations were carried out. Measured average flux of those points is $9.2 \mathrm{mJy}$ for 2005 and $4.2 \mathrm{mJy}$ for 2006. [See the electronic edition of the Journal for a color version of this figure.]

September for $26.0 \mathrm{hr}$, using the so-called wobble mode (Daum et al. 1997), where the object was observed at an $0.4^{\circ}$ offset from the camera center; $25.0 \mathrm{hr}$ of the data passed all quality selection for the analysis. The DISP method was applied for the reconstruction of the shower direction with the final cut on the $\theta^{2}$ parameter (the squared angular distance between the nominal source position and the reconstructed $\gamma$-ray direction). However, no significant excess could be found in the 2006 data.

\section{DISCUSSION}

The Whipple $10 \mathrm{~m}$ telescope observed BL Lacertae for $39.1 \mathrm{hr}$ in 1995 and derived a flux upper limit above $350 \mathrm{GeV}$ at $3.8 \%$ of Crab (Horan et al. 2004). HEGRA derived an upper limit above

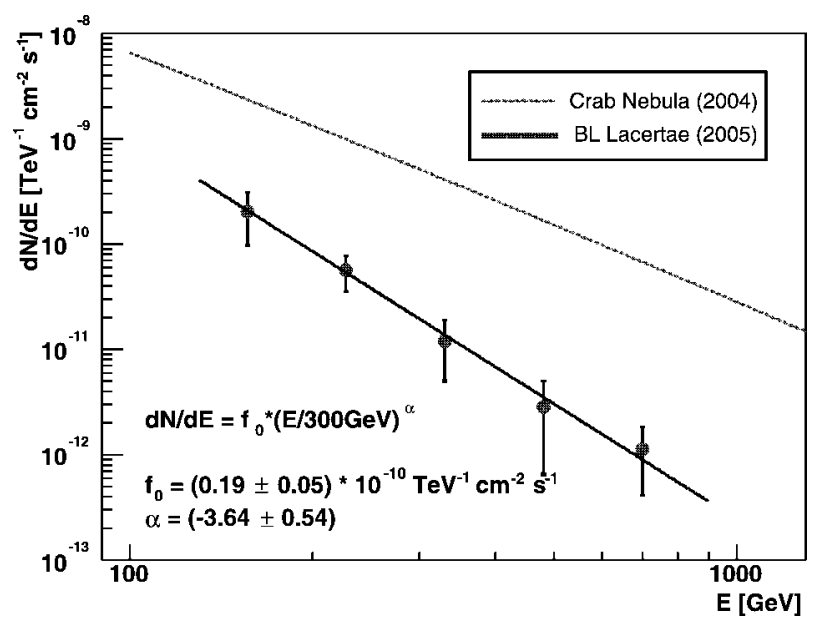

FIG. 4.-Differential energy spectrum of the 2005 BL Lacertae data. The solid line represents a power-law fit to the measured spectrum. The fit parameters are listed in the figure. For comparison, the line fitted to the measured MAGIC Crab spectrum using a power law with a changing photon index is shown by a dashed line (Wagner et al. 2005). [See the electronic edition of the Journal for a color version of this figure.] 
$1.1 \mathrm{TeV}$ at $28 \%$ of Crab with $26.7 \mathrm{hr}$ of observation (Aharonian et al. 2004). These upper limits are consistent with our results. On the other hand, Neshpor et al. (2001) claim VHE $\gamma$-ray detection using the GT 48 telescopes of the Crimean Astrophysical Observatory from data taken in summer 1998. The reported integral $\gamma$-ray flux is $F(E>1 \mathrm{TeV})=(2.1 \pm 0.4) \times 10^{-11} \mathrm{~cm}^{-2} \mathrm{~s}^{-1}$, which is 2 orders of magnitude higher than the extrapolated value from this study. During the same period, 1998 July to August, no significant signal was found by HEGRA, and their reported flux upper limit in the same energy band is 7 times lower than the Crimean result (Kranich 2003). The VHE $\gamma$-ray detection reported by Neshpor et al. cannot be explained without a remarkably huge and a very rapid flux variation offset by a few hours in consecutive nights from the HEGRA observation. In the case of a leptonic origin of the $\gamma$-ray emission, such a flare would possibly coincide with high activity in the optical as in the outburst of 1997 July, when the increase in flux was observed both in the optical and X-ray to $\gamma$-ray bands (Bloom et al. 1997; Tanihata et al. 2000). However, no increased optical activity was detected during the Crimean observation period $\left(m_{p}=13.5-14.6\right.$, while 13.0-14.6 in 2005). Throughout the EGRET observations for BL Lacertae, such a notably huge and rapid flare feature was never reported in the high energy $\gamma$-ray emission, which is considered to have the same origin as the VHE $\gamma$-ray emission regardless of the scenarios for the emission origin.

In Figure 3, filled circles in the optical light curve represent simultaneous observations with the MAGIC telescope, accepting a \pm 1 day offset with respect to the MAGIC observations. In 2005, there are 12 out of 16 nights with simultaneous observations (average flux: $9.2 \mathrm{mJy}$ ), while 16 out of 23 nights in 2006 (average flux: $4.2 \mathrm{mJy}$ ) have coinciding observations. The absence of a significant excess of VHE $\gamma$-rays in the 2006 data indicates that the VHE $\gamma$-ray flux in 2006 was significantly lower than the flux level in 2005. In summary, our results show similar tendencies both in the optical and the VHE $\gamma$-ray flux variations. Similarly, the $\gamma$-ray activity seen by EGRET observations in 1997 showed a strong correlation with optical activity. Such a correlation is expected from leptonic origin scenarios (Bloom et al. 1997). Details of the 2006 data and radio data will be discussed elsewhere.

Figure 5 shows the SED of BL Lacertae with results of this work and some previous data and model calculations by Ravasio et al. (2002). The VHE $\gamma$-ray points are corrected for the extragalactic background light (EBL) absorption using the "low" EBL model of Kneiske et al. (2004). Our optical and VHE $\gamma$-ray points agree well

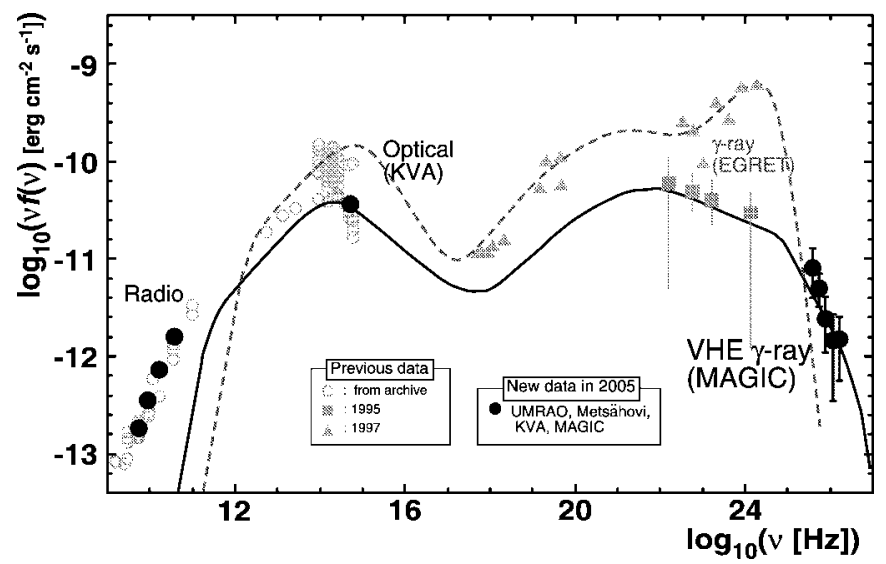

FIG. 5.-SED for BL Lacertae. Black filled circles represent simultaneous 2005 data of KVA and MAGIC as well as radio data taken by UMRAO and Metsähovi. Gray symbols describe old measurements (details in inset). The lines are taken from Ravasio et al. (2002). The solid line represents a one-zone SSC model for 1995 data; the dotted line is produced with SSC and EC components for the 1997 flare data.

with the solid line, which was derived using a one-zone SSC model on the 1995 data, whereas some deviations can be seen from the dotted line, which describes the 1997 flare data and involves SSC as well as EC components (Ravasio et al. 2002). To describe our result such an additional EC component is not necessarily required.

BL Lacertae is the first LBL with a clear detection of VHE $\gamma$ ray emission. The results of this work indicate that VHE $\gamma$-ray observations during times of higher optical states can be more efficient. Future long term monitoring of VHE $\gamma$-ray emission could provide detailed information of a possible periodicity predicted by, e.g., Stirling et al. (2003) and correlations with other wavelengths. Due to the observed steep spectrum, lowering the energy threshold of IACTs (e.g., the upcoming MAGIC-II project) would significantly increase the detection prospects for this new source class.

We thank the IAC for the excellent working conditions at the ORM. The support of the German BMBF and MPG, Italian INFN, and Spanish CICYT is gratefully acknowledged. This work is also supported by ETH Research Grant TH34/043 and the Polish MNiI Grant 1P03D01028. We thank H. Aller and M. Aller (UMRAO), and A. Lähteenmäki (Metsähovi) for providing the radio data.

\section{REFERENCES}

Aharonian, F., et al. 2004, A\&A, 421, 529

Albert, J., et al. 2006, ApJ, 639, 761 2007a, ApJ, 654, L119

2007b, ApJ, 663, 125

2007c, ApJ, in press (astro-ph/0702008)

Baixeras, C., et al. 2004, Nucl. Instrum. Methods Phys. Res. A, 518, 188

Bloom, S. D., et al. 1997, ApJ, 490, L145

Bock, R., et al. 2004, Nucl. Instrum. Methods Phys. Res. A, 516, 511

Breiman, L. 2001, Machine Learning, 45, 5

Bretz, T., et al. 2005, in AIP Conf. Proc. 745, High-Energy Gamma-Ray Astronomy, ed. F. A. Aharonian, H. J. Völk, \& D. Horns (New York: AIP), 730

Catanese, M., et al. 1997, ApJ, 480, 562

Cortina, J., et al. 2005, Proc. 29th Int. Cosmic Ray Conf. (Pune), 5, 359

Daum, A., et al. 1997, Astropart. Phys., 8, 1

Fossati, G., et al. 1998, MNRAS, 299, 433

Gaidos, J., et al. 1996, Nature, 383, 319

Gaug, M., et al. 2005, Proc. 29th Int. Cosmic Ray Conf. (Pune), 5, 375

Ghisellini, G., et al. 1998, MNRAS, 301, 451

Hagen-Thorn, V. A., et al. 1997, Astron. Rep., 41, 154

Hillas, A. M. 1985, Proc. 29th Int. Cosmic Ray Conf. (La Jolla), 3, 445

Horan, D., et al. 2004, ApJ, 603, 51
Knapp, J., \& Heck, D. 2004, EAS Simulation with CORSIKA: A User's Guide (Karlsruhe: Inst. Kernphysik)

Kneiske, T. M., Bretz, T., Mannheim, K., \& Hartman, D. H. 2004, A\&A, 413, 807 Kranich, D. (HEGRA Collaboration). 2003, in ASP Conf. Ser. 299, High Energy Blazar Astronomy, ed. L. O. Takalo \& E. Valtaoja (San Francisco: ASP), 3

Lessard, R. W., et al. 2001, Astropart. Phys., 15, 1

Li, T.-P., \& Ma, Y.-Q. 1983, ApJ, 272, 317

Madejski, G. M., et al. 1999, ApJ, 521, 145

Majumdar, P., et al. 2005, Proc. 29th Int. Cosmic Ray Conf. (Pune), 5, 203

Mannheim, K. 1993, A\&A, 269, 67

Miller, J. S., French, H. B., \& Hawley, S. A. 1978, ApJ, 219, L85

Mücke, A., \& Protheroe, R. J. 2001, Astropart. Phys., 15, 121

Neshpor, Y. I., et al. 1998, Astron. Lett., 24, 134 . 2001, Astron. Rep., 45, 249

Padovani, P., \& Giommi, P. 1995, ApJ, 444, 567

Ravasio, M., et al. 2002, A\&A, 383, 763

Sambruna, R. M., et al. 1999, ApJ, 515, 140

Stirling, A. M., et al. 2003, MNRAS, 341, 405

Tanihata, C., et al. 2000, ApJ, 543, 124

Villata, M., et al. 2004, A\&A, 424, 497

Wagner, R. M., et al. 2005, Proc. 29th Int. Cosmic Ray Conf. (Pune), 4, 163

Wu, X.-B., Liu, F.-K., \& Zhang, T.-Z. 2002, A\&A, 389, 742 